\title{
Alopecia androgenética: $O$ uso do minoxidil e seus efeitos adversos
}

\author{
Androgenic alopecia: The use of minoxidil and it's adverse effects \\ Alopecia androgenética: El uso de minoxidil y sus efectos adversos
}

Recebido: 03/07/2021 | Revisado: 09/07/2021 | Aceito: 12/07/2021 | Publicado: 22/07/2021

\author{
Álvaro Kroetz Grangeiro Gomes \\ ORCID: https://orcid.org/0000-0001-8866-7632 \\ Centro Universitário de Patos, Brasil \\ E-mail: alvarogomes@med.fiponline.edu.br \\ Petrônio Souto Gouveia Filho \\ ORCID: https://orcid.org/0000-0003-2489-5948 \\ Centro Universitário de Patos, Brasil \\ E-mail:petroniofilho@med.fiponline.edu.br
}

\begin{abstract}
Resumo
Objetivo: analisar os efeitos adversos do uso do Minoxidil em pacientes com Alopecia androgenética. Métodos: Foi efetuada uma revisão integrativa da literatura, método que possibilita uma síntese do conhecimento obtido em determinado número de estudos e sua incorporação em forma de aplicabilidade dos resultados obtidos de estudos significativos na prática da pesquisa baseada em evidências. Utilizou-se a questão de pesquisa "Quais são os efeitos adversos do uso de Minoxidil na Alopecia androgenética?". Em seguida foi realizada uma pesquisa no banco de dados internacional Medical Publisher (PUBMED) e na Biblioteca Virtual em Saúde (BVS) com as palavras-chave "Minoxidil" AND "Alopecia" AND "Adverse effects". Resultados: os efeitos adversos mais comuns com o uso do Minoxidil tópico são Hipertricose, prurido no couro cabeludo, dermatite de contato e eritema. Pele seca, aumento da sensibilidade, da produção de caspa também foram relatados. Alguns sintomas menos frequentes foram sinais cardiovasculares sistêmicos, como hipotensão e síncope. Conclusão: O tratamento com Minoxidil tanto em sua conformação tópica quanto sistêmica para a Alopecia Androgenética mostra-se uma medida efetiva e segura para o crescimento capilar, com efeitos adversos podendo ser bem controlados e tolerados.
\end{abstract}

Palavras-chave: Alopecia androgenética; Minoxidil; Reações adversas.

\begin{abstract}
Objective: to analyze the adverse effects of using Minoxidil in patients with androgenic Alopecia. Methods: An integrative literature review was carried out, a method that allows a synthesis of the knowledge obtained in a certain number of studies and its incorporation in the form of applicability of the results obtained from significant studies in the practice of evidence-based research. The research question "What are the adverse effects of using Minoxidil in androgenetic alopecia?" Was used. Then, a search was conducted in the international database Medical Publisher (PUBMED) and in the Virtual Health Library (VHL) with the keywords "Minoxidil" AND "Alopecia" AND "Adverse effects". Results: the most common adverse effects with the use of topical Minoxidil are Hypertrichosis, itchy scalp, contact dermatitis and erythema. Dry skin, increased sensitivity, dandruff production have also been reported. Some less frequent symptoms were systemic cardiovascular signs, such as hypotension and syncope. Conclusion: Treatment with Minoxidil in both it's topical and systemic conformation for Androgenetic Alopecia is an effective and safe measure for hair growth, with adverse effects that can be well controlled and tolerated.
\end{abstract}

Keywords: Androgenetic alopecia; Minoxidil; Adverse reactions.

\section{Resumen}

Objetivo: analizar los efectos adversos del uso de Minoxidil en pacientes con alopecia androgenética. Métodos: Se realizó una revisión integradora de la literatura, método que permite sintetizar los conocimientos obtenidos en un determinado número de estudios y su incorporación en forma de aplicabilidad de los resultados obtenidos de estudios significativos en la práctica de la investigación basada en la evidencia. Se utilizó la pregunta de investigación “¿Cuáles son los efectos adversos del uso de Minoxidil en la Alopecia Androgenética?”. Luego, se realizó una búsqueda en la base de datos internacional Medical Publisher (PUBMED) y en la Biblioteca Virtual en Salud (BVS) con las palabras clave "Minoxidil" Y "Alopecia" Y "Efectos adversos". Resultados: Los efectos adversos más comunes con el uso de Minoxidil tópico son hipertricosis, picazón en el cuero cabelludo, dermatitis de contacto y eritema. También se informó piel seca, aumento de la sensibilidad, aumento de la producción de caspa. Algunos 
síntomas menos frecuentes fueron signos cardiovasculares sistémicos como hipotensión y síncope. Conclusión: El tratamiento con Minoxidil tanto en su configuración tópica como sistémica para la Alopecia Androgenética es una medida eficaz y segura para el crecimiento del cabello, con efectos adversos que pueden ser bien controlados y tolerados.

Palabras clave: Alopecia androgenética; Minoxidil; Reacciones adversas.

\section{Introdução}

Alopecia androgenética, um padrão de perda de cabelo mais frequentemente encontrado no sexo masculino, é uma desordem genética que provoca perda de cabelo mediada pela di-hidrotestosterona, a forma mais potente da testosterona. Essa condição é a causa mais comum de perda de cabelo em ambos os sexos. Esse hormônio, por sua vez, induz degeneração dos folículos pilosos causando transformação do cabelo terminal em cabelo vellus e é o principal mediador da miniaturização capilar e da indução da alopecia androgenética. Sem o tratamento adequado, os pacientes com essa condição genética sofrem com progressiva perda capilar. Essa condição é mais comum nos homens e sua incidência aumenta com a idade (Adil \& Godwin, 2017; Maitra, Goyal \& Rath, 2017; Macedo et al., 2020).

Essa desordem pode provocar distúrbios psicológicos, dentre os quais, privação social, comprometimento da autoestima, preocupaçães ou mesmo negação quanto ao envelhecimento, sensação de desamparo, sentimentos de diminuição da atratividade, além da diminuição da qualidade de vida tanto em homens quanto em mulheres (Adil \& Godwin, 2017; Macedo et al., 2020; Tai \& Kochhar, 2020).

Os pacientes que possuem essa condição perdem o efeito protetor dos cabelos sobre os raios ultravioletas, temperaturas muito frias e trauma físico. Além disso, os mesmos possuem maior risco de desenvolvimento de câncer de pele na região sem cabelo como resultado da patogênese desse distúrbio (Tai \& Kochhar, 2020).

Muitos fatores, ambientais, endócrinos e genéticos, possuem papel fundamental na patogênese dessa condição. O crescimento capilar se inicia na fase anágena, na qual o folículo começa a se desenvolver e crescer, sendo mediado por muitas citocinas. Um aumento na concentração de andrógenos nos folículos pilosos induz a um encurtamento no ciclo de crescimento capilar e cabelos cada vez mais finos e curtos. $\mathrm{O}$ fator de crescimento semelhante a insulina (IGF-1) tem demonstrado ter forte capacidade de induzir crescimento capilar e inibir um estado catágeno nos folículos pilosos (Macedo et al., 2019; Barat, Abdollahimajd, Dadkhahfar \& Moravvej, 2020; Macedo et al., 2020).

O Minoxidil é atualmente utilizado como tratamento de primeira linha para a Alopecia androgenética em solução a $2 \%$ e 5\%. Requer duas aplicações diárias e com duração de pelo menos 4 horas na região da pele aplicada. Quando em contato com a pele, o Minoxidil é convertido a sulfato de Minoxidil, que potencializa os canais de potássio no músculo liso, provocando seu relaxamento. Assim, aumenta-se a perfusão no folículo piloso, estendendo a fase anágena e estimulando o crescimento folicular, diminuindo assim a conversão do cabelo terminal para cabelo vellus (Macedo et al., 2020; Tai \& Kochhar, 2020).

Os principais e mais relevantes efeitos adversos do uso do Minoxidil tópico são dermatite de contato, taquicardia e hipertricose. A irritação na pele é menos proeminente com a solução a $2 \%$. Cuidados devem ser tomados com o uso da solução tópica, sendo eles: evitar deixar resíduos da solução em lençóis, e travesseiros que possam entrar em contato com face, olhos e boca, sendo que a aplicação horas antes de dormir diminui muito esse risco. Pacientes com doença cardiovascular devem usar o medicamento com cuidado devido à taquicardia documentada pelo seu uso (Tai \& Kochhar, 2020).

A forma oral do Minoxidil também apresenta alguns efeitos adversos semelhantes, dentre os quais, prurido, irritação na pele, hipertricose, sendo este o efeito mais comum observado, retenção de sódio e de fluidos provocando hipervolemia, podendo provocar edema generalizado e ganho de peso, devendo ser evitado ou mesmo utilizado com cautela em pacientes com insuficiência cardíaca ou doença renal, mesmo esses efeitos sendo pouco comuns (Randolph \& Tosti, 2017). 
Dessa forma, esse estudo busca analisar, a partir de uma revisão integrativa da literatura, os efeitos adversos do uso do Minoxidil em pacientes com Alopecia androgenética. Dentre as razões para justificar a realização desse tipo de estudo, importante em temáticas atuais e ainda pouco abordadas em pesquisas científicas, destaca-se a possibilidade de contribuir com informações relevantes para as práticas clínicas e ao meio científico, fornecendo evidências científicas seguras na íntegra para aplicação em campo em populações portadoras de alopecia androgenética.

\section{Metodologia}

Foi efetuada uma revisão integrativa da literatura, método que possibilita uma síntese do conhecimento obtido em determinado número de estudos e sua incorporação em forma de aplicabilidade dos resultados obtidos de estudos significativos na prática da pesquisa baseada em evidências. Em virtude de sua abordagem metodológica, esse método permite a inclusão de diversos métodos, com o objetivo de desempenhar um importante papel na pesquisa baseada em evidências, permitindo a inclusão de estudos experimentais e não-experimentais para uma completa compreensão do problema analisado na pesquisa. Além disso, ela combina dados da literatura teórica e empírica, além de incorporar uma ampla gama de objetivos: definição de conceitos, revisão de teorias e evidências, e análise de problemas metodológicos de um tópico particular (Souza, Silva \& Carvalho, 2010).

A revisão integrativa da literatura consegue identificar o atual conhecimento sobre um problema específico e atual, uma vez que é realizada de modo a pontuar, analisar e sintetizar resultados de estudos independentes sobre o mesmo assunto, contribuindo dessa forma para uma possível repercussão benéfica na prática clínica e na qualidade dos cuidados prestados à população. Assim, pontua-se que o impacto da utilização da revisão integrativa é essencial para o desenvolvimento de políticas, protocolos e procedimento além de possibilitar a disseminação de informações atuais e relevantes para a prática diária (Souza, Silva \& Carvalho, 2010).

A seguir, destacam-se as principais etapas para a sua realização: 1 - Identificação do tema e seleção da questão de pesquisa, 2 - estabelecimento dos critérios de inclusão e exclusão, 3 - identificação dos estudos pré-selecionados e selecionados, 4 - categorização dos estudos selecionados, 5 - análise e interpretação dos resultados e 6 - apresentação da revisão/síntese do conhecimento (Souza, Silva \& Carvalho, 2010).

Inicialmente foi realizada a escolha do tema, relacionado com ALOPECIA ANDROGENÉTICA e a partir daí, definiu-se o título "Alopecia androgenética: o uso do Minoxidil e seus efeitos adversos", e em um segundo momento foi escolhida a questão de pesquisa "Quais são os efeitos adversos do uso de Minoxidil na Alopecia androgenética?".

Em seguida foi realizada uma pesquisa no banco de dados internacional Medical Publisher (PUBMED) e na Biblioteca Virtual em Saúde (BVS) com as palavras-chave "Minoxidil" AND "Alopecia" AND "Adverse effects".

Foram aplicados critérios de inclusão e exclusão, objetivando diminuir amostragem final de artigos relacionados ao tema tais como: artigos publicados nos últimos cinco anos, excluindo-se artigos repetidos. A partir dessas etapas realizadas foram identificadas 285 publicações e selecionados 16 artigos (Figura 1) com o uso de critérios de inclusão e exclusão. 
Figura 1: Etapas seguidas para seleção dos artigos que compõem esse estudo conforme recomendação PRISMA (Galvão, 2015).

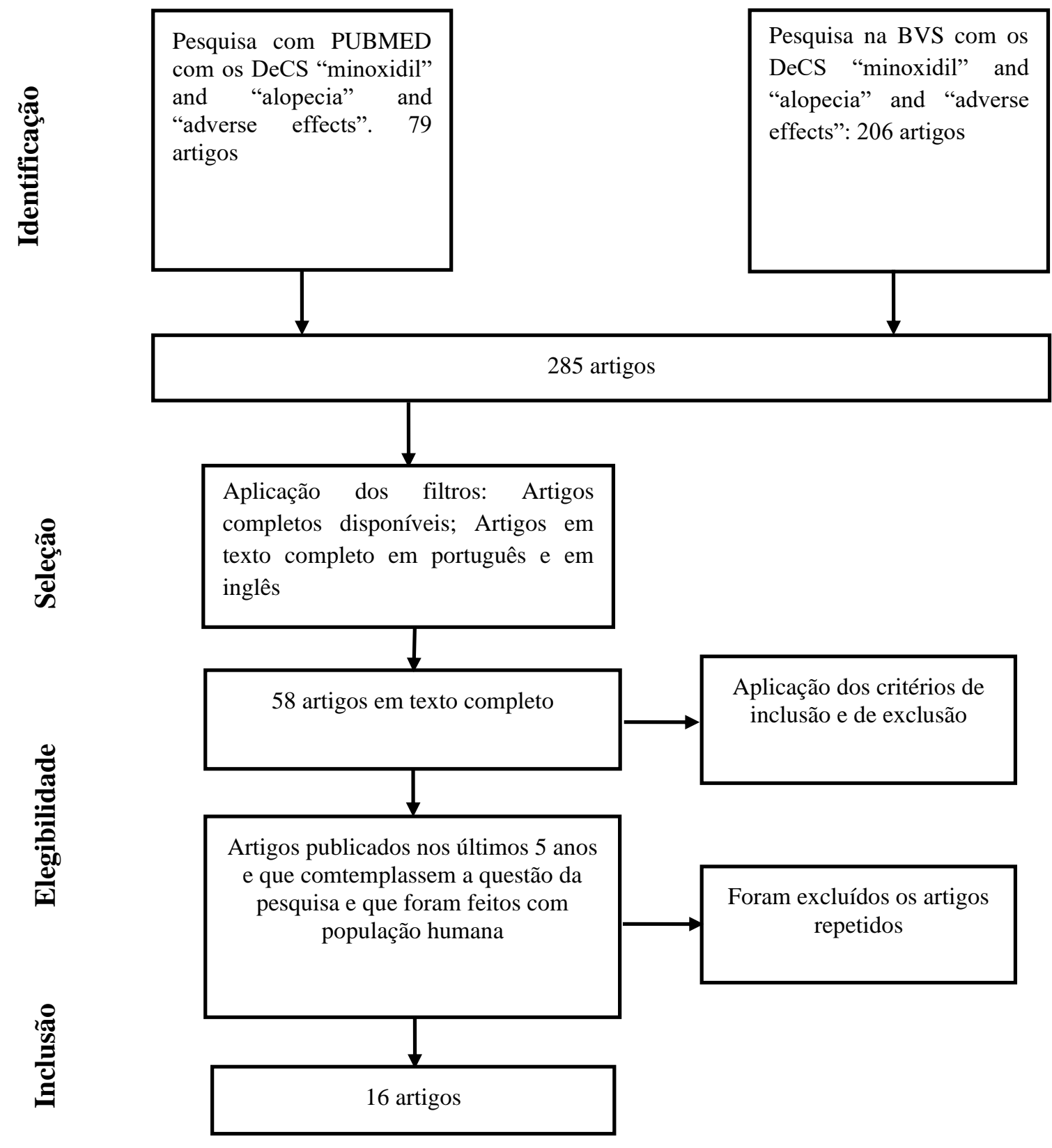

Fonte: Autores (2021).

Posteriormente, foram extraídas as seguintes informações: autor, ano de publicação da pesquisa, título e país de origem para formar os artigos da amostra final. Os artigos foram agrupados em categorias: Minoxidil e Alopecia androgenética.

\section{Resultados}

De acordo com as informações do Quadro 1, é possível observar que a revista de publicação mais frequente foi a Dermatologic Therapy com $(\mathrm{N}=3)$ publicações. A amostra de 16 publicações subdivide-se entre 11 países diferentes que publicaram sobre o tema nos últimos 5 anos. Quinze publicações estão em inglês e apenas uma em espanhol. 
Quadro 1: Caracterização dos artigos de acordo com autores, título, base de dados, jornal de publicação e país de origem.

\begin{tabular}{|c|c|c|c|c|}
\hline Autor (ano) & Título do artigo & $\begin{array}{c}\text { Base de } \\
\text { dados }\end{array}$ & $\begin{array}{l}\text { Revista de } \\
\text { publicação }\end{array}$ & País \\
\hline Gong et al. (2017) & $\begin{array}{l}\text { The clinical effect of JetpPeel-assisted } \\
\text { topical minoxidil in the treatment of } \\
\text { androgenetic alopecia: A randomized } \\
\text { pilot study }\end{array}$ & BVS & $\begin{array}{l}\text { Journal of Cosmetic } \\
\text { and Laser Therapy }\end{array}$ & China \\
\hline $\begin{array}{l}\text { Mckesey \& Pandya, } \\
\text { (2019) }\end{array}$ & $\begin{array}{l}\text { Effectiveness and safety of low-dose } \\
\text { oral minoxidil in male androgenetic } \\
\text { alopecia }\end{array}$ & BVS & $\begin{array}{l}\text { Journal Of The } \\
\text { American Academy } \\
\text { Of Dermatology }\end{array}$ & $\begin{array}{l}\text { Estados } \\
\text { Unidos da } \\
\text { América } \\
\end{array}$ \\
\hline $\begin{array}{lr}\text { Ramos, } & \text { Sinclair, } \\
\text { Kasprzak, \& } \text { Miot } \\
(2020)\end{array}$ & $\begin{array}{l}\text { Minoxidil } 1 \mathrm{mg} \text { oral versus minoxidil } \\
5 \% \text { topical solution for the treatment of } \\
\text { female-pattern hair loss: A randomized } \\
\text { clinical trial }\end{array}$ & BVS & $\begin{array}{l}\text { Journal Of The } \\
\text { American Academy } \\
\text { Of Dermatology }\end{array}$ & Brasil \\
\hline Stoehr et al. (2019) & $\begin{array}{l}\text { Off-Label Use of Topical Minoxidil in } \\
\text { Alopecia: A Review }\end{array}$ & BVS & $\begin{array}{l}\text { American Journal of } \\
\text { Clinical Dermatology }\end{array}$ & Suíça \\
\hline $\begin{array}{l}\text { Suchonwanit, } \\
\text { Rojhirunsakool } \quad \& \\
\text { Khunkhet (2019) }\end{array}$ & $\begin{array}{l}\text { A randomized, investigator-blinded, } \\
\text { controlled, split-scalp study of the } \\
\text { efficacy and safety of a 1550-nm } \\
\text { fractional erbium-glass laser, used in } \\
\text { combination with topical 5\% minoxidil } \\
\text { versus 5\% Minoxidil alone, for the } \\
\text { treatment of androgenetic alopecia }\end{array}$ & BVS & $\begin{array}{l}\text { Lasers in Medical } \\
\text { Science }\end{array}$ & Tailândia \\
\hline Rampon et al. (2016) & $\begin{array}{l}\text { Infantile generalized hypertrichosis } \\
\text { caused by topical minoxidil }\end{array}$ & PUBMED & $\begin{array}{l}\text { Anais Brasileiros de } \\
\text { Dermatologia }\end{array}$ & Brasil \\
\hline $\begin{array}{ll}\text { Panchaprateep } & \& \\
\text { Lueangarun }(2020) & \end{array}$ & $\begin{array}{l}\text { Efficacy and Safety of Oral Minoxidil } \\
5 \mathrm{mg} \text { Once Daily in the Treatment of } \\
\text { Male Patients with Androgenetic } \\
\text { Alopecia: An Open-Label and Global } \\
\text { Photographic Assessment }\end{array}$ & PUBMED & $\begin{array}{l}\text { Dermatology And } \\
\text { Therapy }\end{array}$ & Tailândia \\
\hline $\begin{array}{lr}\text { Van } & \text { Zuuren; } \\
\text { Fedorowicz; Schoones } \\
(2016)\end{array}$ & $\begin{array}{l}\text { Interventions for female pattern hair loss } \\
\text { (Review) }\end{array}$ & PUBMED & $\begin{array}{l}\text { Cochrane Database of } \\
\text { Systematic Reviews }\end{array}$ & Holanda \\
\hline $\begin{array}{l}\text { Suchonwanit, } \\
\text { Thammarucha \& \& } \\
\text { Leerunyakul (2019) }\end{array}$ & $\begin{array}{l}\text { Minoxidil and its use in hair disorders: a } \\
\text { review }\end{array}$ & PUBMED & $\begin{array}{l}\text { Drug Design, } \\
\text { Development And } \\
\text { Therapy }\end{array}$ & Talândia \\
\hline $\begin{array}{l}\text { Beach, Renée } \quad \text { A. } \\
(2018)\end{array}$ & $\begin{array}{l}\text { Case series of oral minoxidil for } \\
\text { androgenetic and traction alopecia: } \\
\text { Tolerability \& the five C's of oral } \\
\text { therapy }\end{array}$ & PUBMED & $\begin{array}{l}\text { Dermatologic } \\
\text { Therapy }\end{array}$ & Canadá \\
\hline $\begin{array}{l}\text { Dubrey, } \\
\text { VanGriethuysen } \quad \& \\
\text { Edwards, (2015) }\end{array}$ & $\begin{array}{l}\text { A hairy fall: syncope resulting from } \\
\text { topical application of Minoxidil }\end{array}$ & PUBMED & $\begin{array}{l}\text { British Medical } \\
\text { Journal }\end{array}$ & Reino Unido \\
\hline Gargallo et al., (2015) & $\begin{array}{l}\text { Generalized Hypertrichosis Due to } \\
\text { Topical Minoxidil }\end{array}$ & PUBMED & $\begin{array}{l}\text { Actas Dermo- } \\
\text { Sifiliográficas }\end{array}$ & Espanha \\
\hline Hu et al., (2015) & $\begin{array}{l}\text { Combined treatment with oral } \\
\text { finasteride and topical minoxidil in male } \\
\text { androgenetic alopecia: a randomized and } \\
\text { comparative study in Chinese patients }\end{array}$ & PUBMED & $\begin{array}{l}\text { Dermatologic } \\
\text { Therapy }\end{array}$ & China \\
\hline Wu, Yu \& Li (2016) & $\begin{array}{l}\text { Differences in reproductive toxicology } \\
\text { between alopecia drugs: an analysis on } \\
\text { adverse events among female and male } \\
\text { cases }\end{array}$ & PUBMED & Oncotarget & China \\
\hline Rossi et al. (2016) & $\begin{array}{l}\text { Multi-therapies in } \begin{array}{r}\text { androgenetic } \\
\text { alopecia: review and clinical } \\
\text { experiences }\end{array} \\
\end{array}$ & PUBMED & $\begin{array}{l}\text { Dermatologic } \\
\text { Therapy }\end{array}$ & Itália \\
\hline $\begin{array}{l}\text { Cranwell \& Sinclair } \\
(2016)\end{array}$ & Male Androgenetic Alopecia & PUBMED & $\begin{array}{l}\text { National Institutes of } \\
\text { Health }\end{array}$ & Austrália \\
\hline
\end{tabular}


A partir da análise do Quadro 2, pode-se inferir que os efeitos adversos mais comuns com o uso do Minoxidil tópico são Hipertricose, prurido no couro cabeludo, dermatite de contato e eritema. Pele seca, aumento da sensibilidade, da produção de caspa também foram relatados. Alguns sintomas menos frequentes foram sinais cardiovasculares sistêmicos, como hipotensão e síncope. Ambos esses sintomas cessam com o tratamento é interrompido.

Quadro 2: Objetivos dos estudos selecionados e principais resultados encontrados pelos autores.

\begin{tabular}{|c|c|c|}
\hline Autor (ano) & Objetivo do estudo & Principais efeitos adversos do uso do Minoxidil \\
\hline Gong et al. (2017) & $\begin{array}{l}\text { Uso do Minoxidil tópico associado com } \\
\text { Jet Peel para observar se há aceleração } \\
\text { do crescimento capilar. }\end{array}$ & $\begin{array}{l}\text { Hipertricose, dermatite de contato e prurido no couro } \\
\text { cabeludo. }\end{array}$ \\
\hline $\begin{array}{l}\text { Mckesey e Pandya, } \\
\text { (2019) }\end{array}$ & $\begin{array}{l}\text { Avaliar a efetividade e segurança do } \\
\text { uso de Minoxidil oral em baixas doses } \\
(2,5-5 \mathrm{mg}) \text { em homens com alopecia } \\
\text { androgenética. }\end{array}$ & $\begin{array}{l}\text { Os efeitos adversos mais comuns foram hipertricose, edema } \\
\text { de membros inferiores e queda de cabelo na fase inicial do } \\
\text { tratamento. Uma pequena parcela apresentou alterações } \\
\text { eletrocardiográficas. Ambos os efeitos foram leves e bem } \\
\text { tolerados pelos pacientes. }\end{array}$ \\
\hline $\begin{array}{l}\text { Ramos, Sinclair, } \\
\text { Kasprzak, \& Miot } \\
(2020)\end{array}$ & $\begin{array}{l}\text { Comparar a eficácia do Minoxidil oral } \\
\text { com o tópico no tratamento de alopecia } \\
\text { feminina. }\end{array}$ & $\begin{array}{l}\text { Os efeitos adversos mais frequentes com o uso do Minoxidil } \\
\text { oral foram hipertricose, edema de membros inferiores, } \\
\text { aumento da frequência cardíaca e aumento da pressão } \\
\text { arterial. Já com sua composição tópica, Hipertricose e } \\
\text { prurido no couro cabeludo foram os mais comuns, porém } \\
\text { aumento da frequência cardíaca e da pressão arterial também } \\
\text { estiveram presentes. }\end{array}$ \\
\hline Stoehr et al. (2019) & $\begin{array}{l}\text { Descrever e avaliar o uso do Minoxidil } \\
\text { tópico para alopecia androgenética. }\end{array}$ & $\begin{array}{l}\text { Hipertricose facial, dermatite de contato e queda de cabelo } \\
\text { no primeiro mês de uso, alteração na pressão arterial e } \\
\text { tontura, foram os efeitos adversos mais relatados. }\end{array}$ \\
\hline $\begin{array}{l}\text { Suchonwanit; } \\
\text { Rojhirunsakool; } \\
\text { Khunkhet (2019) }\end{array}$ & $\begin{array}{l}\text { Comparação entre o uso do laser erbium } \\
\text { glass associado com o Minoxidil tópico } \\
5 \% \text { versus Minoxidil 5\% isolado. }\end{array}$ & $\begin{array}{l}\text { Eritema, irritação, prurido no couro cabeludo, erosão e } \\
\text { cabelos quebradiços no início do tratamento foram os mais } \\
\text { relatados. }\end{array}$ \\
\hline $\begin{array}{l}\text { Rampon et al. } \\
(2016)\end{array}$ & $\begin{array}{l}\text { Apresentar o primeiro relato de caso no } \\
\text { Brasil de uma criança de } 5 \text { anos com } \\
\text { Hipertricose generalizada após uso de } \\
\text { Minoxidil tópico. }\end{array}$ & Hipertricose generalizada, taquicardia e palpitações. \\
\hline $\begin{array}{l}\text { Panchaprateep; } \\
\text { Lueangarun (2020) }\end{array}$ & $\begin{array}{l}\text { Avaliar a eficácia e a segurança do uso } \\
\text { do Minoxidil oral uma vez ao dia para } \\
\text { tratamento de alopecia androgenética } \\
\text { masculina. }\end{array}$ & Hipertricose e edema de membros inferiores. \\
\hline $\begin{array}{l}\text { Van Zuuren; } \\
\text { Fedorowicz; } \\
\text { Schoones (2016) }\end{array}$ & $\begin{array}{l}\text { Avaliar a eficácia e segurança dos } \\
\text { tratamentos disponíveis para tratamento } \\
\text { da perda de cabelo feminino. }\end{array}$ & $\begin{array}{l}\text { Prurido no couro cabeludo, irritação na pele, dermatite de } \\
\text { contato, pele seca e Hipertricose. }\end{array}$ \\
\hline $\begin{array}{l}\text { Suchonwanit; } \\
\text { Thammarucha; } \\
\text { Leerunyakul } \\
\text { (2019) }\end{array}$ & $\begin{array}{l}\text { Revisar e atualizar informações clínicas } \\
\text { sobre o uso do Minoxidil tópico. }\end{array}$ & $\begin{array}{l}\text { Hipertricose, queda de cabelo no primeiro mês de uso, } \\
\text { prurido no couro cabeludo, dermatite de contato, palpitaça, } \\
\text { taquicardia e alteração na pressão arterial. }\end{array}$ \\
\hline $\begin{array}{l}\text { BEACH, Renée A. } \\
\text { (2018) }\end{array}$ & $\begin{array}{l}\text { Detalhar a tolerabilidade do uso do } \\
\text { Minoxidil oral para o tratamento da } \\
\text { alopecia androgenética. }\end{array}$ & $\begin{array}{l}\text { Hipertricose, alterações na pressão sanguínea, dermatite de } \\
\text { contato, prurido no couro cabeludo e sintomas de } \\
\text { hipotensão. }\end{array}$ \\
\hline $\begin{array}{l}\text { Dubrey; } \\
\text { VanGriethuysen; } \\
\text { Edwards, (2015) }\end{array}$ & $\begin{array}{l}\text { Relatar um caso de um jovem que } \\
\text { apresentou efeitos cardiovasculares } \\
\text { sistêmicos, como hipotensão } \\
\text { significante e síncope com uso de } \\
\text { Minoxidil tópico. }\end{array}$ & $\begin{array}{l}\text { Pré-síncope ao fazer esforços e síncope após exercícios mais } \\
\text { intensos. }\end{array}$ \\
\hline $\begin{array}{l}\text { Gargallo et al. } \\
(2015)\end{array}$ & $\begin{array}{l}\text { Relatar um caso de hipertricose } \\
\text { generalizada em mulher após uso de } \\
\text { Minoxidil tópico. }\end{array}$ & Prurido no couro cabeludo e aumento da sensibilidade. \\
\hline Hu et al. (2015) & $\begin{array}{l}\text { Comparar a eficácia da monoterapia e a } \\
\text { terapia combinada de Minoxidil 5\% e } \\
\text { finasterida. }\end{array}$ & $\begin{array}{l}\text { Dermatite de contato foi o efeito adversos mais comum com } \\
\text { o uso de Minoxidil tópico, seguido por prurido no couro } \\
\text { cabeludo, sensação de queimação, dor de cabeça e } \\
\text { Hipertricose. }\end{array}$ \\
\hline $\mathrm{Wu}$; Yu; Li, (2016) & $\begin{array}{l}\text { Relatar os principais efeitos adversos } \\
\text { com o uso do Minoxidil. }\end{array}$ & $\begin{array}{l}\text { Disfunção erétil, depressão, prurido no couro cabeludo, } \\
\text { diminuição da libido, hipoestesia, ansiedade, dor de cabeça, } \\
\text { dermatite de contato, tontura e corioretinopatia foram os } \\
\text { efeitos mais comuns nos homens. Rosto inchado, dermatite } \\
\text { de contato, artralgia, palpitações, tontura, náusea, }\end{array}$ \\
\hline
\end{tabular}




\begin{tabular}{|l|l|l|}
\hline & & $\begin{array}{l}\text { taquicardia, ganho de peso, prurido no couro cabeludo e } \\
\text { diminuição da acuidade visual foram os efeitos mais comuns } \\
\text { nas mulheres. }\end{array}$ \\
\hline Rossi et al. (2016) & $\begin{array}{l}\text { Analisar as terapias existentes para o } \\
\text { tratamento da alopecia androgenética. }\end{array}$ & $\begin{array}{l}\text { Hipertricose em outras regiões do corpo que não o couro } \\
\text { cabeludo, prurido na cabeça, eritema, pele seca e dermatite } \\
\text { de contato. }\end{array}$ \\
\hline $\begin{array}{l}\text { Cranwell e Sinclair } \\
(2016)\end{array}$ & $\begin{array}{l}\text { Relatar os principais efeitos adversos no } \\
\text { tratamento para alopecia androgenética. }\end{array}$ & $\begin{array}{l}\text { Hipertricose, aumento de caspa e eritema no couro cabeludo, } \\
\text { queda de cabelo no primeiro mês de uso são os efeitos } \\
\text { adversos mais comuns com uso do Minoxidil tópico. }\end{array}$ \\
\hline
\end{tabular}

Fonte: Autores (2021).

De acordo com os principais achados dos efeitos adversos do uso de Minoxidil tópico, pode-se afirmar que a Hipertricose (75\%) e prurido no couro cabeludo (68,75\%) foram os mais comuns com o uso dessa apresentação. Enquanto que os efeitos adversos sistêmicos mais comuns com Minoxidil oral foram alterações na pressão arterial (37,5\%) e aumento da frequência cardíaca junto com palpitações $(18,75 \%)$ logo em seguida.

Quadro 3: Caracterização dos artigos conforme categoria e subcategoria, de acordo com os efeitos adversos tópicos e sistêmicos mais frequentes.

\begin{tabular}{|c|c|c|c|}
\hline Categorias & Subcategorias & Quantidade & Porcentagem (\%) \\
\hline \multirow{4}{*}{$\begin{array}{l}\text { Efeitos adversos } \\
\text { tópicos }\end{array}$} & Hipertricose & 12 & 75,00 \\
\hline & Prurido no couro cabeludo & 11 & 68,75 \\
\hline & Dermatite de contato & 8 & 50,00 \\
\hline & Cabelos quebradiços no início do tratamento & 5 & 31,25 \\
\hline \multirow{5}{*}{$\begin{array}{l}\text { Efeitos adversos } \\
\text { sistêmicos }\end{array}$} & Alterações na pressão arterial & 6 & 37,5 \\
\hline & Aumento da frequência cardíaca/palpitações & 3 & 18,75 \\
\hline & Síncope e pré-síncope & 2 & 12,50 \\
\hline & Edema de membros inferiores & 2 & 12,50 \\
\hline & Alterações cardíacas & 2 & 12,50 \\
\hline
\end{tabular}

Fonte: Autores (2021).

\section{Discussão}

O uso do Minoxidil em longo prazo promove um avanço mais rápido do fio capilar para a fase telógena, porém ajuda o mesmo fio a entrar em uma fase anágena mais nova e saudável, promovendo assim o crescimento do fio capilar e prologando a fase anágena, evitando assim a queda de cabelo (Gong, Bao, Tian \& Li 2018).

O tratamento com Minoxidil oral mostrou trazer benefícios ao tratamento clínico de pacientes com alopecia androgenética, retardando a queda de cabelo. No entanto, alguns efeitos adversos foram observados, tais como hipertricose, edema de membros inferiores e a queda de cabelo na fase inicial do tratamento. Tais efeitos foram bem tolerados pelos pacientes, não sendo necessário a interrupção do tratamento (Gargallo, Gutierrez, Vanaclocha \& Guerra-Tapia, 2015; Cranwell \& Sinclair, 2016; Mckesey \& Pandya, 2019). 
O uso do Minoxidil oral em longo prazo tem demonstrado ser capaz de aumentar a densidade total do cabelo, apesar de trazer alguns efeitos adversos, tais como prurido no couro cabeludo, edema de membros inferiores, hipertricose facial e aumento da frequência cardíaca, porém sem alteração na pressão arterial. Todos esses efeitos foram bem tolerados e não foi preciso a cessação do tratamento (Gargallo, Gutierrez, Vanaclocha \& Guerra-Tapia, 2015; Cranwell \& Sinclair, 2016; Rossi et al., 2016; Panchaprateep \& Lueangarun 2020; Ramos, Sinclair, Kasprzak, \& Miot 2020).

Alguns dos parâmetros de eficácia que podem ser levados em consideração e relação ao tratamento com o Minoxidil podem ser a contagem dos fios capilares na área aplicada, diâmetro da área calva ao longo do tratamento e avaliação feita tanto pelo paciente quanto pelo médico. $\mathrm{O}$ mecanismo pelo qual o Minoxidil promove o crescimento do cabelo ainda não está bem esclarecido. Em alguns estudos de seu mecanismo de ação, foi demonstrado que a medicação aumento o fluxo sanguíneo para a região do couro cabeludo, além de promover a transição dos folículos capilares da fase telógena de seu repouso até uma fase anágena ativa pela ativação dos canais de potássio. Os efeitos adversos mais comumente relatados com o uso da droga incluem a hipertricose facial, dermatite de contato e queda de cabelo transitória no primeiro mês de uso (Hu et al., 2015; Cranwell \& Sinclair, 2016; Stoehr, Choi, Colavincenzo \& Vanderweil, 2019; Ramos, Sinclair, Kasprzak, \& Miot 2020).

O Minoxidil é absorvido pela pele por difusão normal, sendo que massagens na aplicação ou fricção facilitam a absorção, a partir daí é convertido no seu metabólito ativo, sulfato de Minoxidil, pela sulfotransferase, uma enzima presente na região do couro cabeludo humano. O provável mecanismo para crescimento capilar é o aumento da regulação dos níveis dos fatores de crescimento do endotélio vascular e da prostaglandina E2, assim como um aumento do fluxo sanguíneo na região do couro cabeludo. Os efeitos adversos observados com o tratamento do Minoxidil tópico foram eritema, prurido e erosão capilar (Wu, Yu, Li, 2016; Suchonwanit, Rojhirunsakool \& Khunkhet, 2019; Suchonwanit, Thammarucha \& Leerunyakul, 2019).

Os principais possíveis mecanismos que justificam o mecanismo de ação do Minoxidil no crescimento capilar são ação na vasculatura e na síntese de DNA das células foliculares, estimulando seu crescimento com efeito agonista nos canais de potássio sensíveis ao ATP e prolongamento da fase anágena folicular (Rampon, Henkin, Souza \& Almeida, 2016; Wu, Yu $\& \mathrm{Li}, 2016)$.

Hipertricose secundária ao uso do Minoxidil tópico é mais comum nas mulheres do que nos homens. A absorção sistêmica da droga é tipicamente mínima em sua conformação tópica, porém a absorção varia entre as pessoas, o que pode explicar a taquicardia e as palpitações que são relatadas em alguns pacientes que aplicam a medicação diretamente na pele. Isso pode sugerir que é possível o Minoxidil tópico alcançar concentrações mais elevadas no plasma de alguns indivíduos, explicando alguns efeitos sistêmicos relatados (Rampon, Henkin, Souza \& Almeida, 2016).

$\mathrm{O}$ tratamento com Minoxidil oral aumenta significativamente o crescimento capilar, a densidade, o diâmetro do cabelo e diminui o ritmo de queda de cabelo. Baixa dose oral desse medicamento pode ser considerada uma modalidade terapêutica segura para os pacientes saudáveis, com poucos efeitos adversos sendo relatados, tais como hipertricose e edema de membros inferiores (Panchaprateep \& Lueangarun, 2020).

Seu uso promove uma mudança para a fase anágena prematura e prolonga o tempo que os fios capilares permanecem nessa fase. Ocorre assim o encurtamento da fase telógena com aceleração dessa fase, levando a uma sincronização do ciclo capilar e um aumento da queda de cabelo no início da terapia com Minoxidil. Perda essa que é apenas temporária, pois o fio está apenas retornando para uma fase anágena que durará mais tempo (Panchaprateep \& Lueangarun, 2020).

Os efeitos colaterais sistêmicos mais graves relatados com o uso do Minoxidil oral são complicações cardiovasculares, mais comuns em pacientes com doença cardíaca, mas que também podem acontecer em pacientes sem essa condição. Doença cardíaca isquêmica, pericardite, derrame pericárdico e tamponamento pulmonar, hipertensão e insuficiência cardíaca de alto débito foram as condições mais observadas. A angina pode piorar ou surgir pela primeira vez durante o 
tratamento com Minoxidil, provavelmente sendo justificado devido ao aumento da demanda de oxigênio associado com o aumento da frequência cardíaca (Panchaprateep \& Lueangarun, 2020).

Devido a essas condições e como medidas de segurança, o tratamento com Minoxidil oral deve ser desencorajado e desaconselhado em pacientes com fatores de risco cardiovascular, tais como indivíduos idosos com risco aumentado de infarto agudo do miocárdio, insuficiência cardíaca, doença renal crônica ou hipertensão grave. Essa modalidade terapêutica deve ser considerada apenas para indivíduos saudáveis (Panchaprateep \& Lueangarun, 2020).

Com a descontinuação do tratamento, todo o crescimento capilar atingido com as aplicações pode ser perdido e espera-se a reincidência de queda de cabelo em até três meses e todo os efeitos benéficos aos folículos pilosos terão desaparecido em até seis meses (Van Zuuren, Fedorowicz \& Schoones, 2016).

Os efeitos adversos mais comuns observados com o Minoxidil tópico 5\% foram hipertricose, dermatite de contato com irritação, prurido no couro cabeludo, descamação e dores de cabeça. A cautela que se deve ter com o Minoxidil tópico é que o excesso de aplicação tópica dessa droga pode induzir absorção sistêmica, levando ao crescimento de pelo em áreas indesejadas. Aplicação duas vezes ao dia dessa composição não leva a efeitos adversos sistémicos, tais como alterações na frequência cardíaca, hipotensão e ganho de peso (Rossi et al., 2016; Beach, 2018; Suchonwanit, Thammarucha \& Leerunyakul, 2019).

Efeitos adversos sistêmicos graves, tais como distúrbios do sódio, retenção de líquidos e efeitos cardiovasculares, tais como doença isquêmica do coração, derrame pericárdico e hipertensão pulmonar) foram relatados com a administração oral de Minoxidil. A retenção de sódio e de líquidos leva a congestão sistêmica e edema de membros inferiores, favorecendo a ocorrência de insuficiência cardíaca (Suchonwanit, Thammarucha \& Leerunyakul, 2019).

Efeitos sistêmicos como pré-síncope e síncope são raros de serem observados no tratamento tópico do Minoxidil, entretanto, quando usado em dosagens muito elevadas e por mecanismo já descrito anteriormente, pode gerar episódios de tontura e até desmaios, dependendo da atividade física sendo realizada pelo paciente (Dubrey, Vangriethuysen \& Edwards, 2015; Wu, Yu \& Li, 2016).

\section{Considerações Finais}

O tratamento com Minoxidil tanto em sua conformação tópica quanto sistêmica para a Alopecia Androgenética (AAG) mostra-se uma medida efetiva e segura para o crescimento capilar nesses pacientes, com efeitos adversos podendo ser bem controlados e tolerados.

Os efeitos adversos mais comumente relatados e observados nos pacientes que fazem uso do Minoxidil tópico foram hipertricose facial, prurido no couro cabeludo, dermatite de contato e cabelos quebradiços no primeiro mês de tratamento. Já os efeitos adversos sistêmicos mais comuns foram alterações na pressão arterial, taquicardia e palpitações, pré-síncope, síncope, tontura e edema de membros inferiores. Devido ao seu mecanismo de ação, a modalidade oral não deve ser prescrita para pacientes com aumento do risco cardiovascular devido ao risco de complicações cardíacas.

Sugere-se para estudos futuros uma melhor elucidação sobre a herança genética que predispõe à Alopecia Androgenética e os mecanismos moleculares que resultam na queda de cabelo. Além disso, pesquisas para avaliar melhor medicamentos que consigam agir de maneira segura apenas nos genes relevantes para AAG são necessárias para ampliar as opções farmacológicas para o tratamento adequado e eficaz desse distúrbio.

\section{Referências}

Adil, A., \& Godwin, M. (2017). The effectiveness of treatments for androgenetic alopecia: A systematic review and meta-analysis. Journal of the American Academy of Dermatology, 77(1), 136-141.e5. https://doi.org/10.1016/j.jaad.2017.02.054 
Barat, T., Abdollahimajd, F., Dadkhahfar, S., \& Moravvej, H. (2020). Evaluation of the efficacy and safety of cow placenta ex tract lotion versus minoxidil $2 \%$ in the treatment of female pattern androgenetic alopecia. International journal of women's dermatology, 6(4), 318-321. https://doi.org/10.1016/j.ijwd.2020.04.012

Beach R. A. (2018). Case series of oral minoxidil for androgenetic and traction alopecia: Tolerability \& the five C's of oral therapy. Dermatologic therapy, 31(6), e12707. https://doi.org/10.1111/dth.12707

Cranwell, W. \& Sinclair R. (2016). Male Androgenetic Alopecia. Endotext [Internet]. https://www.ncbi.nlm.nih.gov/books/NBK278957/

Dubrey, S. W., VanGriethuysen, J., \& Edwards, C. M. (2015). A hairy fall: syncope resulting from topical application of minoxidil. BMJ case reports, bcr2015210945. https://doi.org/10.1136/bcr-2015-210945

Galvão, T F. (2015). Principais itens para relatar Revisões sistemáticas e Meta-análises: a recomendação prisma. Epidemiologia e Serviços de Saúde, 24(2), 335-342. http://dx.doi.org/10.5123/s1679-49742015000200017.

Gargallo, V., Gutierrez, C., Vanaclocha, F., \& Guerra-Tapia, A. (2015). Hipertricosis generalizada secundaria a minoxidil tópico. Actas Dermo-Sifiliográficas, 106(7), 599-600. http://dx.doi.org/10.1016/j.ad.2014.12.016.

Gong, L., Bao, L., Tian, T., \& Li, Y. (2018). The clinical effect of JetpPeel-assisted topical minoxidil in the treatment of androgenetic alopecia: A randomized pilot study. Journal of cosmetic and laser therapy: official publication of the European Society for Laser Dermatology, 20(1), 58-63. https://doi.org/10.1080/14764172.2017.1341046.

Hu, R., Xu, F., Sheng, Y., Qi, S., Han, Y., Miao, Y., Rui, W., \& Yang, Q. (2015). Combined treatment with oral finasteride and topical minoxidil in male androgenetic alopecia: a randomized and comparative study in Chinese patients. Dermatologic therapy, 28(5), 303-308. https://doi.org/10.1111/dth.12246.

Jimenez-Cauhe, J., Saceda-Corralo, D., Rodrigues-Barata, R., Hermosa-Gelbard, A., Moreno-Arrones, O. M., Fernandez-Nieto, D., \& Vaño-Galvan, S. (2019). Effectiveness and safety of low-dose oral minoxidil in male androgenetic alopecia. Journal of the American Academy of Dermatology, 81(2), 648-649. https://doi.org/10.1016/j.jaad.2019.04.054

Macedo, C. L. A. de., Santana, T. S., Ferreira, A. F. P., Jucá, N. B. H., Crispim, K. S. L, Banhos, M. E. H., Amorim, L. C. P., Rabelo, K. P. S., Brasil, D. R. M., Basso, S., Araújo, R. C. de., Rios, M. S., Costa, A. J. A. da., \& Castro Júnior, J. E. di. (2020). Androgenetic alopecia (New capillary protocol in androgenic alopecia). Research, Society and Development, 9(9), e989997457. https://doi.org/10.33448/rsd-v9i9.7457

Macedo, J. L., Oliveira, A. S. da S. S., Pereira, I. C., Assunção, F. D., Reis, E. R., \& Assunção, M. de J. S. M. (2019). Efficacy of phytotherapy in the treatment of androgenetic alopecia. Research, Society and Development, 8(5), e285868. https://doi.org/10.33448/rsd-v8i5.868

Maitra, M., Goyal, A. K., \& Rath, G. (2017). A novel approach for follicular delivery of minoxidil for treatment of alopecia. Journal Of Drug Delivery Science And Technology, 41(17) 113-123. http://dx.doi.org/10.1016/j.jddst.2017.07.002.

Panchaprateep, R., \& Lueangarun, S. (2020). Efficacy and Safety of Oral Minoxidil 5 mg Once Daily in the Treatment of Male Patients with Androgenetic Alopecia: An Open-Label and Global Photographic Assessment. Dermatology and therapy, 10(6), 1345-1357. https://doi.org/10.1007/s13555-020-00448-x

Ramos, P. M., Sinclair, R. D., Kasprzak, M., \& Miot, H. A. (2020). Minoxidil $1 \mathrm{mg}$ oral versus minoxidil 5\% topical solution for the treatment of femalepattern hair loss: A randomized clinical trial. Journal of the American Academy of Dermatology, 82(1), 252-253. https://doi.org/10.1016/j.jaad.2019.08.060

Rampon, G., Henkin, C., de Souza, P. R., \& Almeida, H. L., Jr (2016). Infantile generalized hypertrichosis caused by topical minoxidil. Anais brasileiros de dermatologia, 91(1), 87-88. https://doi.org/10.1590/abd1806-4841.20164010

Randolph, M., \& Tosti, A. (2021). Oral minoxidil treatment for hair loss: A review of efficacy and safety. Journal of the American Academy of Dermatology, 84(3), 737-746. https://doi.org/10.1016/j.jaad.2020.06.1009

Rossi, A., Anzalone, A., Fortuna, M. C., Caro, G., Garelli, V., Pranteda, G., \& Carlesimo, M. (2016). Multi-therapies in androgenetic alopecia: review and clinical experiences. Dermatologic therapy, 29(6), 424-432. https://doi.org/10.1111/dth.12390

Souza, M. T. de, Silva, M. D. da, Carvalho, R. de (2010). Integrative review: what is it? how to do it? Einstein (São Paulo), 8(1), 102-106. http://dx.doi.org/10.1590/s1679-45082010rw1134.

Stoehr, J. R., Choi, J. N., Colavincenzo, M., \& Vanderweil, S. (2019). Off-Label Use of Topical Minoxidil in Alopecia: A Review. American journal of clinical dermatology, 20(2), 237-250. https://doi.org/10.1007/s40257-018-0409-y

Suchonwanit, P., Rojhirunsakool, S., \& Khunkhet, S. (2019). A randomized, investigator-blinded, controlled, split-scalp study of the efficacy and safety of a 1550-nm fractional erbium-glass laser, used in combination with topical 5\% minoxidil versus 5\% minoxidil alone, for the treatment of androgenetic alopecia. Lasers in medical science, 34(9), 1857-1864. https://doi.org/10.1007/s10103-019-02783-8

Suchonwanit, P., Thammarucha, S., \& Leerunyakul, K. (2019). Minoxidil and its use in hair disorders: a review. Drug design, development and therapy, 13, 2777-2786. https://doi.org/10.2147/DDDT.S214907

Tai, T., \& Kochhar, A. (2020). Physiology and Medical Treatments for Alopecia. Facial plastic surgery clinics of North America, 28(2), 149-159. https://doi.org/10.1016/j.fsc.2020.01.004

Van Zuuren, E. J., Fedorowicz, Z., \& Schoones, J. (2016). Interventions for female pattern hair loss. The Cochrane database of systematic reviews, 2016(5), CD007628. https://doi.org/10.1002/14651858.CD007628.pub4

Wu, M., Yu, Q., \& Li, Q. (2016). Differences in reproductive toxicology between alopecia drugs: an analysis on adverse events among female and male cases. Oncotarget, 7(50), 82074-82084. https://doi.org/10.18632/oncotarget.12617 